\title{
Description of poly(ethylenepropylene) confined in nanopores by a modified Rouse model
}

Matthias Muthmann, Margarita Krutyeva, Lutz Willner, Jürgen Allgaier, Dieter Richter, Reiner Zorn, Michael Ohl, Vivian Rebbin, and Peter Lindner

Citation: The Journal of Chemical Physics 146, 203309 (2017); doi: 10.1063/1.4975977

View online: http://dx.doi.org/10.1063/1.4975977

View Table of Contents: http://aip.scitation.org/toc/jcp/146/20

Published by the American Institute of Physics

\section{Articles you may be interested in}

Polymer dynamics under cylindrical confinement featuring a locally repulsive surface: A quasielastic neutron scattering study

The Journal of Chemical Physics 146, 203306 (2017); 10.1063/1.4974836

Local glass transition temperature $\mathrm{T}_{\mathrm{g}}(\mathrm{z})$ of polystyrene next to different polymers: Hard vs. soft confinement The Journal of Chemical Physics 146, 203307 (2017); 10.1063/1.4975168

Glassy dynamics of polymethylphenylsiloxane in one- and two-dimensional nanometric confinement-A comparison

The Journal of Chemical Physics 146, 203302 (2017); 10.1063/1.4974767

Reduced-mobility layers with high internal mobility in poly(ethylene oxide)-silica nanocomposites

The Journal of Chemical Physics 146, 203303 (2017); 10.1063/1.4974768

Unexpected impact of irreversible adsorption on thermal expansion: Adsorbed layers are not that dead The Journal of Chemical Physics 146, 203304 (2017); 10.1063/1.4974834

Relaxation processes and glass transition of confined polymer melts: A molecular dynamics simulation of 1,4polybutadiene between graphite walls

The Journal of Chemical Physics 146, 203308 (2017); 10.1063/1.4975390

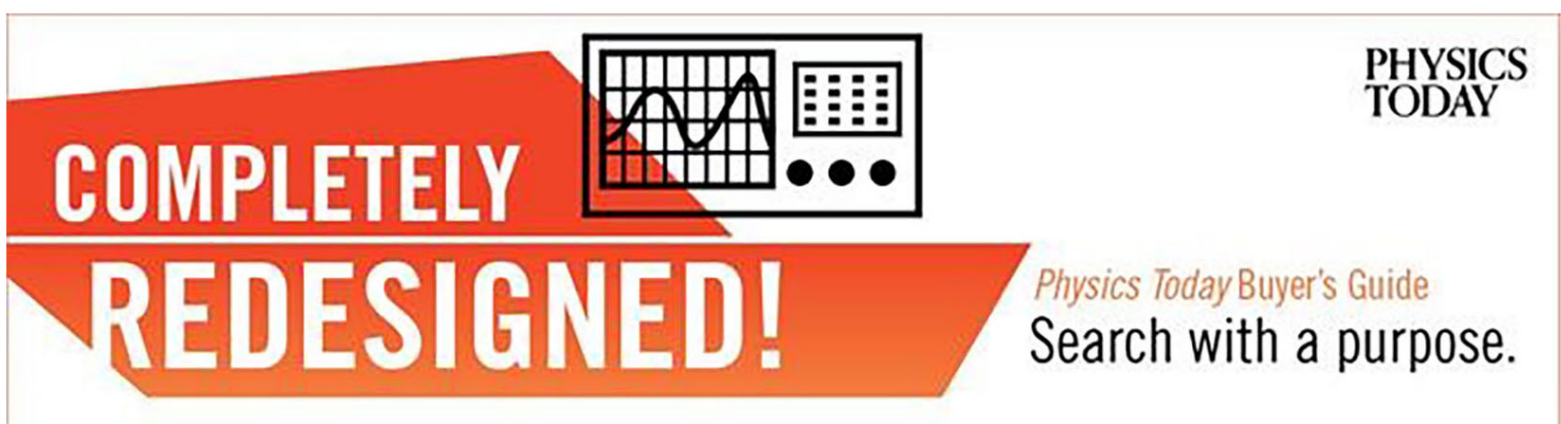




\title{
Description of poly(ethylenepropylene) confined in nanopores by a modified Rouse model
}

\author{
Matthias Muthmann, ${ }^{1}$ Margarita Krutyeva, ${ }^{1}$ Lutz Willner, ${ }^{1}$ Jürgen Allgaier, ${ }^{1}$ Dieter Richter, ${ }^{1}$ \\ Reiner Zorn, ${ }^{1}$ Michael Ohl, ${ }^{2}$ Vivian Rebbin, ${ }^{3}$ and Peter Lindner ${ }^{4}$ \\ ${ }^{1}$ JCNS-1, Forschungszentrum Jülich, D-52425 Jülich, Germany \\ ${ }^{2}$ JCNS Outstation at SNS, Forschungszentrum Jülich, Oak Ridge, Tennessee 37831, USA \\ ${ }^{3}$ HASYLAB DESY, Notkestr. 85, D-22607 Hamburg, Germany \\ ${ }^{4}$ Institut Laue-Langevin, B.P. 156, F-38042 Grenoble, France
}

(Received 1 December 2016; accepted 27 January 2017; published online 15 February 2017)

A recent model for unentangled polymer chains in confinement [M. Dolgushev and M. Krutyeva, Macromol. Theory Simul. 21, 565 (2012)] is scrutinized by small-angle neutron scattering (SANS) with respect to its static prediction, the single-chain structure factor. We find a remarkable agreement although the model simplifies the effect of the confinement to a harmonic potential. The effective confinement size from fits of SANS data with the model agrees well with the actual pore size. Starting from this result we discuss the possibility of an experiment on the dynamic structure factor predicted by the model. It turns out that such an experiment would need a large ratio polymer dimension/pore size which is difficult but not impossible to achieve. Published by AIP Publishing. [http://dx.doi.org/10.1063/1.4975977]

\section{INTRODUCTION}

The structure and dynamics of polymers in confinement on a length scale comparable to the chain dimension has recently gained much interest. ${ }^{1}$ One reason for this is that such a situation can be found in applications as nanocomposites but also in biology in the so-called crowded environment of a cell. The other reason is rooted in the theoretical interest in a description of the confined chain analogous to that of the free chain by the Rouse model, reptation model, and similar approaches. In spite of this growing interest, there are yet not many theoretical descriptions present. Furthermore, if calculations exist, as that of Freed et al. for the structure of a chain between interacting walls, ${ }^{2}$ those are often too complicated to be evaluated for the practical description of data.

In this paper, we will use a comparatively simple model by Dolgushev and Krutyeva ${ }^{3}$ (DK model) for a polymer chain confined in a cylinder. This model consists of a variation of the Rouse model by adding a cylindrically symmetric harmonic potential. The DK model has two parameters, the radius of gyration of the unperturbed chain $R_{\mathrm{g} 0}$ and a force constant of dimension length, $d$. Due to the choice of a harmonic potential, the Rouse modes are still eigenmodes of the system and only the mode relaxation times and amplitudes are changed. The result of the DK model is the dynamic structure factor $S(Q, t)$. As a special case $t=0$, the result comprises the structure factor $S(Q)$ of a single chain. Although an analytical solution is not possible (as it is in the case of the Rouse model for the unperturbed chain), the numerical calculation is rather simple involving a summation over pairs of Rouse modes.

\section{RECAP OF CURRENT THEORIES}

The dynamics of non-entangled polymer chains in the melt is usually well described by the Rouse model. ${ }^{4}$ This model is based on Langevin equations for the motion of $N$ beads connected by (entropic) springs,

$$
\zeta \frac{\mathrm{d} \mathbf{r}_{n}}{\mathrm{~d} t}=\frac{3 k_{\mathrm{B}} T}{l^{2}}\left(\mathbf{r}_{n+1}-2 \mathbf{r}_{n}+\mathbf{r}_{n-1}\right)+\mathbf{f}_{n}(t) .
$$

In this equation $\zeta$ is the monomeric friction coefficient and $\mathbf{f}_{n}(t)$ a random force. The fluctuations of the random force are related to the friction coefficient by $\left\langle f_{n \alpha}(t) f_{m \beta}\left(t^{\prime}\right)\right\rangle$ $=2 \zeta k_{\mathrm{B}} T \delta_{m n} \delta_{\alpha \beta} \delta\left(t-t^{\prime}\right)$. The spring force constant is fixed due to its entropic nature to $3 k_{\mathrm{B}} T / l^{2}$, where $l^{2}$ is the mean-square distance between beads. The system of Equation (1) can be decoupled by introducing so-called Rouse modes $\mathbf{X}_{p}(t)$,

$$
\mathbf{r}_{n}(t)=\mathbf{X}_{0}(t)+2 \sum_{p=1}^{N-1} \mathbf{X}_{p}(t) \cos \left(\frac{\pi p n}{N}\right) .
$$

Solving the equations of motions for the Rouse modes, summing over all modes, and summing over all pairs of beads lead to the coherent scattering function (a.k.a dynamic structure factor) of the chain,

$$
\begin{aligned}
S(Q, t)= & \frac{1}{N} \sum_{m, n=1}^{N} \exp \left(-Q^{2}\left(D t+\frac{l^{2}}{6}|n-m|\right.\right. \\
& \left.\left.+4 \sum_{p=1}^{N-1}\left\langle X_{p}^{2}\right\rangle \cos \left(\frac{\pi p m}{N}\right) \cos \left(\frac{\pi p n}{N}\right)\left(1-\mathrm{e}^{-t / \tau_{p}}\right)\right)\right),
\end{aligned}
$$

where the (center-of-mass) diffusion coefficient $D$, the mode amplitudes $\left\langle X_{p}^{2}\right\rangle$, and the mode relaxation times $\tau_{p}$ all follow from the microscopic parameters of the model,

$$
\begin{aligned}
D & =\frac{k_{\mathrm{B}} T}{N \zeta}, \\
\left\langle X_{p}^{2}\right\rangle & =\left(\frac{24 N}{l^{2}} \sin ^{2}\left(\frac{\pi p}{2 N}\right)\right)^{-1},
\end{aligned}
$$




$$
\tau_{p}=\left(\frac{12 k_{\mathrm{B}} T}{\zeta l^{2}} \sin ^{2}\left(\frac{\pi p}{2 N}\right)\right)^{-1}
$$

For the practical application, it is often preferred to use the mean-square end-to-end distance $R_{\mathrm{e}}^{2}=N l^{2}$ and the "Rouse rate" $W l^{4}=3 k_{\mathrm{B}} T l^{2} / \zeta$ as parameters. The reason is that with these parameters the results of the Rouse model, in particular (3), become independent of the number of beads $N$ used in the description of the chain in the limit of large $N$.

The static structure factor corresponds to the limit $S(Q)$ $=S(Q, 0)$. Then, Equation (3) simplifies to

$$
S(Q)=\frac{1}{N} \sum_{m, n=1}^{N} \exp \left(-\frac{Q^{2} l^{2}}{6}|n-m|\right) .
$$

In the limit of large $N$, the structure factor can be expressed in closed form as the Debye function,

$$
S(Q)=2 N \frac{\exp \left(-R_{\mathrm{g}}^{2} Q^{2}\right)-1+R_{\mathrm{g}}^{2} Q^{2}}{R_{\mathrm{g}}^{4} Q^{4},}
$$

where $R_{\mathrm{g}}^{2}=R_{\mathrm{e}}^{2} / 6$ is the squared radius of gyration.

In order to describe a polymer chain in confinement, one has to add a force resulting from a potential to the Langevin equation. If one would choose a "realistic" potential, e.g., a hard cylinder wall, an analytical solution would in most cases be impossible. Therefore, the DK model introduces the confinement of the polymer to a cylindrical channel simply by adding a two-dimensional harmonic potential,

$$
\begin{aligned}
\zeta \frac{\mathrm{d} \mathbf{r}_{n}}{\mathrm{~d} t}= & \frac{3 k_{\mathrm{B}} T}{l^{2}}\left(\mathbf{r}_{n+1}-2 \mathbf{r}_{n}+\mathbf{r}_{n-1}\right) \\
& -\nabla\left(\frac{k_{\mathrm{B}} T}{N d^{2}} \sum\left(x_{n}^{2}+y_{n}^{2}\right)\right)+\mathbf{f}_{n}(t) .
\end{aligned}
$$

The additional parameter $d$ determines the strength of the confinement. $d=\infty$ would correspond to the unconfined chain and $d=0$ to the chain strictly confined to the $z$ axis. Although $d$ has the dimension of length, it cannot be directly identified as the confinement size. Finally, it should be noted that this choice of $d$ also results in an independence of $N$ for large $N$. Note that the definition of $d$ here is different from that of $\tilde{d}$ in Ref. 3, namely, $d=\tilde{d} / 2 \sqrt{N}$. Because the total potential is still harmonic, the Rouse modes (2) are still solutions, but their amplitudes and relaxation times are modified in the directions perpendicular to the axis,

$$
\begin{aligned}
\left\langle X_{1,2 p}^{2}\right\rangle & =\left(\frac{24 N}{l^{2}} \sin ^{2}\left(\frac{\pi p}{2 N}\right)+\frac{4}{d^{2}}\right)^{-1}, \\
\left\langle X_{3 p}^{2}\right\rangle & =\left(\frac{24 N}{l^{2}} \sin ^{2}\left(\frac{\pi p}{2 N}\right)\right)^{-1}, \\
\tau_{1,2 p} & =\left(\frac{12 k_{\mathrm{B}} T}{\zeta l^{2}} \sin ^{2}\left(\frac{\pi p}{2 N}\right)+\frac{2 k_{\mathrm{B}} T}{N \zeta d^{2}}\right)^{-1}, \\
\tau_{3 p} & =\left(\frac{12 k_{\mathrm{B}} T}{\zeta l^{2}} \sin ^{2}\left(\frac{\pi p}{2 N}\right)\right)^{-1} .
\end{aligned}
$$

Here, the additional index denotes the Cartesian components, 1 and 2 are those perpendicular to the axis, and 3 is parallel to the axis. Also the diffusion coefficient is modified into a perpendicular value and a (unchanged) parallel,

$$
\begin{aligned}
& D_{\perp}=\frac{d^{2}}{2 t}\left(1-\exp \left(-\frac{2 k_{\mathrm{B}} T}{N \zeta d^{2}} t\right)\right), \\
& D_{\|}=\frac{k_{\mathrm{B}} T}{N \zeta} .
\end{aligned}
$$

In contrast to the unconfined diffusion coefficient (4) (which is still valid for the unconfined direction parallel to the axis), the diffusion coefficient in the perpendicular directions decays with time. For short times it is equal to the parallel/unconfined value, $\lim _{t \rightarrow 0} D_{\perp}(t)=D_{\|}$. For long times $D_{\perp}$ decays inverse proportionally with $t$. This compensates the $t$-proportionality of the mean square displacement of the center of mass in Equation (3) and leads to a constant value $\lim _{t \rightarrow \infty}\left\langle x_{\mathrm{com}}^{2}(t)\right\rangle$ $=\lim _{t \rightarrow \infty} 2 D_{\perp} t=d^{2}$. This corresponds to the intuitive picture that the diffusive motion starts without "feeling" the walls and finally gets "stuck" limited by the confinement size.

The calculation of the coherent scattering function is complicated by the fact that the amplitudes of the Rouse modes, their decay times, and the diffusion constant are anisotropic. A direct application of (3) leads to a vector-dependent $S(\mathbf{Q}, t)$. Because in the experiments here the pores are orientationally disordered, this result has to be averaged over all orientations of Q. This calculation has been done by Dolgushev and Krutyeva with the result

$S(Q, t)=\frac{\sqrt{\pi}}{2 N} \sum_{m, n=1}^{N} \frac{\operatorname{erf}\left(Q \sqrt{B_{m n}-A_{m n}}\right)}{Q \sqrt{B_{m n}-A_{m n}}} \exp \left(-A_{m n} Q^{2}\right)$

with

$$
\begin{aligned}
A_{m n}= & D_{\perp} t+\sum_{p=1}^{N-1}\left\langle X_{1 p}^{2}\right\rangle\left(2\left(\cos \left(\frac{\pi p m}{N}\right)-\cos \left(\frac{\pi p n}{N}\right)\right)^{2}\right. \\
& \left.+4 \cos \left(\frac{\pi p m}{N}\right) \cos \left(\frac{\pi p n}{N}\right)\left(1-\mathrm{e}^{-t / \tau_{1 p}}\right)\right)
\end{aligned}
$$

and

$$
\begin{aligned}
B_{m n}= & D_{\|} t+\sum_{p=1}^{N-1}\left\langle X_{3 p}^{2}\right\rangle\left(2\left(\cos \left(\frac{\pi p m}{N}\right)-\cos \left(\frac{\pi p n}{N}\right)\right)^{2}\right. \\
& \left.+4 \cos \left(\frac{\pi p m}{N}\right) \cos \left(\frac{\pi p n}{N}\right)\left(1-\mathrm{e}^{-t / \tau_{3 p}}\right)\right) .
\end{aligned}
$$

Note that the simplifications which are possible for the Rouse model which lead to a closed form result in the limit of large $N$ are not possible here. Therefore the sums have to be calculated numerically, and the computational effort increases with $N^{3}$. Using pre-calculated arrays for $\tau_{\alpha p}$ and $\cos (\pi n m / N)$ allows the calculation of these sums for $N=100$ in reasonable time to be used as fit functions on a personal computer.

The calculation is greatly simplified if only the static structure factor is to be calculated. Equation (16) still holds but with $A_{m n}$ and $B_{m n}$ replaced by

$$
A_{m n}^{0}=2 \sum_{p=1}^{N-1}\left\langle X_{1 p}^{2}\right\rangle\left(\cos \left(\frac{\pi p m}{N}\right)-\cos \left(\frac{\pi p n}{N}\right)\right)^{2}
$$

and

$$
B_{m n}^{0}=2 \sum_{p=1}^{N-1}\left\langle X_{3 p}^{2}\right\rangle\left(\cos \left(\frac{\pi p m}{N}\right)-\cos \left(\frac{\pi p n}{N}\right)\right)^{2} .
$$


Again, the simplifications which lead to the Debye function in the case of the unmodified Rouse model for $N \rightarrow \infty$ are not possible for expression (19).

The (squared) radius of gyration in directions perpendicular to the axis is ${ }^{3}$

$$
R_{\mathrm{g} 1,2}^{2}=\frac{d^{2}}{4}\left(\sqrt{\frac{2}{3}} \frac{R_{\mathrm{e}}}{d} \operatorname{coth}\left(\sqrt{\frac{2}{3}} \frac{R_{\mathrm{e}}}{d}\right)-1\right)
$$

with the root-mean-square end-to-end distance of the unperturbed chain, $R_{\mathrm{e}}=\sqrt{N} l$, in the limit of large $N$, while that in parallel direction remains unchanged,

$$
R_{\mathrm{g} 3}^{2}=R_{\mathrm{g} 0}^{2} / 3=R_{\mathrm{e}}^{2} / 18 .
$$

As expected, the limit of (21) for the unconfined chain is $\lim _{d \rightarrow \infty} R_{\mathrm{g} 1,2}^{2}=N l^{2} / 18$ while that for strong confinement is $\lim _{d \rightarrow 0} R_{\mathrm{g} 1,2}^{2}=R_{\mathrm{e}} d / \sqrt{24}$. It has to be noted that the latter limit shows that even for strong confinement the radius of gyration in the confined directions is not just given by $d$ but still depends on that of the unconfined chain proportionally to its square root. This counter-intuitive result arises from the competition between the entropic force on the chain favoring a size $\sim R_{\mathrm{e}}$ and the external potential of the confinement favoring a size $\sim d$ leading to a "compromise" which is the geometric mean of both length scales.

Applying the DK model to the realistic situation of a polymer confined to a cylindrical channel, it is tempting to identify $d$ with the pore radius $R_{\text {cyl }}$ or at least to a value proportional to it. While for $d \gg R_{\mathrm{e}}$ this is correct, the competition of length scales mentioned above leads to a different result if both lengths are of the same order. A comparison of the mean square distance of the monomers from the axis of the channel, which is $\left\langle\rho^{2}\right\rangle=R_{\text {cyl }}^{2} / 2$ in the case of a cylinder, with the value calculated in Ref. 5 leads to

$$
R_{\text {cyl }}=d \sqrt{\frac{R_{\mathrm{e}}}{\sqrt{6} d} \operatorname{coth}\left(\sqrt{\frac{2}{3}} \frac{R_{\mathrm{e}}}{d}\right)+\frac{3}{2}} .
$$

This equation will be used to compare $d$ with the pore dimension throughout this paper. Its limits for weak and strong confinement are $\lim _{d \rightarrow \infty} R_{\text {cyl }}=\sqrt{2} d$ and $\lim _{d \rightarrow 0} R_{\text {cyl }}=6^{-1 / 4} \sqrt{R_{\mathrm{e}} d}$, respectively.

Fig. 1 shows the comparison between the Rouse and the DK models. It can be seen that the DK model causes a "hump" appearing in the scattering curve. This is due to an incipient transition from the power law $Q^{-2}$ of the Debye function to that of a linear scatterer, $Q^{-1}$. ${ }^{5}$ It can also be seen that comparatively small values of $d / R_{\mathrm{e}}$ are necessary to make the effect visible at all. The deviation between the Rouse model and the Debye function at $R_{\mathrm{g}} Q \gtrsim 10$ originates from the finite number of beads considered $(N=100)$. The calculated curve (also for the DK model) levels off at $S(Q)=1$ while the Debye function decays until infinite $Q$ because infinite $N$ is assumed. Of course, the scattering from a real (finite) polymer chain will also level off when $1 / Q$ reaches the dimension of the monomer. So the structure factors calculated from the sums will probably represent reality better although they do not capture the inner structure of the monomer correctly.

For the fit of the bulk polymer data, a correction was applied due to the different molecular masses and the high

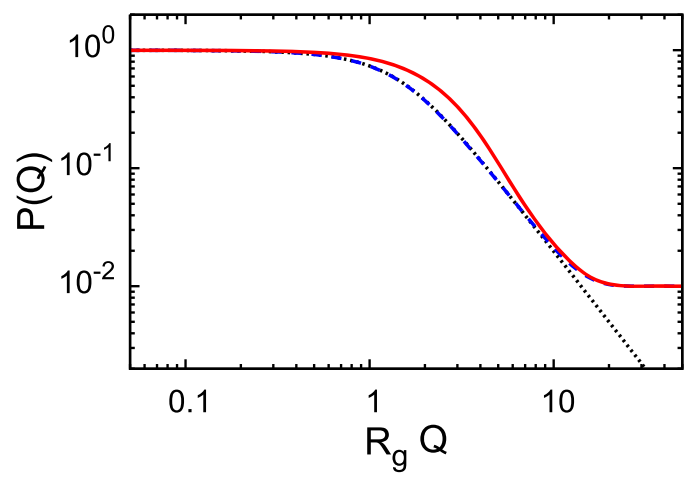

FIG. 1. Comparison of the static form factor $P(Q)=S(Q) / N$ of a polymer chain from the Rouse model (blue, dashed) and the DK model (red). For comparison the Debye function is shown as a dotted line. The deviation between the Rouse model and the Debye function at high $Q$ originates from the finite number of beads considered $(N=100)$. For the DK model $d / R_{\mathrm{e}}=0.081$ was chosen which is the value resulting from the experiments on $50 \mathrm{k}$ poly(ethylene-propylene) (PEP).

content of protonated polymer. The normalized SANS intensity was fitted using the random phase approximation (RPA) for mixtures,

$$
I(Q)=\Delta \varrho^{2}\left(\frac{1}{\phi V_{\mathrm{ph}} P_{\mathrm{h}}(Q)}+\frac{1}{(1-\phi) V_{\mathrm{pd}} P_{\mathrm{d}}(Q)}-2 \chi_{\mathrm{vol}}\right)^{-1},
$$

where $P_{\mathrm{h}}(Q)$ and $P_{\mathrm{d}}(Q)$ are the form factors of the protonated and deuterated polymers, respectively $(P(Q)=S(Q) / N$, thus $P(0)=1$ independent of $N) . V_{\mathrm{ph}}$ and $V_{\mathrm{pd}}$ are the molar volumes. Both quantities are different for the protonated and deuterated polymers because of the different molecular masses. $\phi$ is the volume fraction of the protonated polymer and $\chi_{\text {vol }}=6.8 \times 10^{-6} \mathrm{~mol} / \mathrm{cm}^{3}$ is the Flory-Huggins parameter taken from the literature. ${ }^{6} \Delta \varrho$ is the difference of the scattering length densities of the deuterated and protonated material per mole. This correction contributes significantly $(>1 \%)$ only for the 50k sample for the lowest $Q$ values $<0.03 \AA^{-1}$. There are additional uncertainties for the confinement samples (e.g., degree of filling) which do not allow to apply this correction. But since the differences between Rouse and DK model take place at higher $Q$, this correction could be neglected for the confined materials.

\section{EXPERIMENTAL}

\section{A. Samples}

For the experimental test of the DK model, we used a polymer in cylindrical pores where the materials are chosen in a way to minimize the interaction between both components. As confining material, we used SBA- $15,{ }^{7}$ a type of nanoporous silica synthesized by a templating technique based on triblock copolymers. ${ }^{8}$ As can be seen from Fig. 2 , the pores are a hexagonal array of cylinders. Although the pores are oriented within the grains of micrometer size, the sample is macroscopically isotropic because of the random orientation of the grains. From the decrease of small-angle neutron scattering (SANS) upon filling the pores with a polymer, one can estimate that the pores constitute $50 \%$ of the total SBA-15 volume. Together with a pore distance of $105 \AA$ from the first Bragg peak in SANS, this results in a radius of the pores of $39 \AA$. 


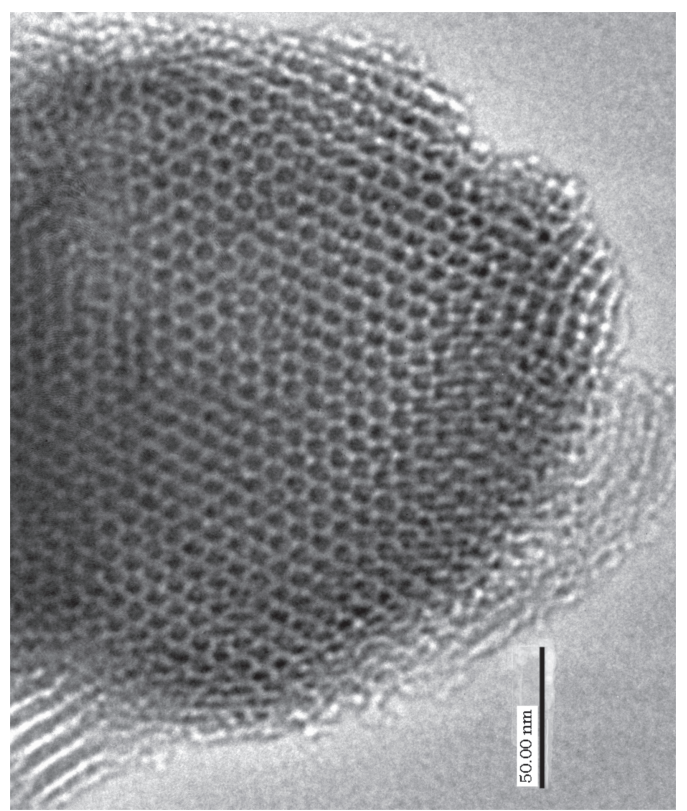

FIG. 2. Transmission electron microscopy of the SBA-15 used in this study. The scale bar indicates $500 \AA$.

The pores were filled with atactic poly(ethylenepropylene) (PEP). Mixtures of PEP with natural isotopic composition and deuterated PEP were used to make the single chain properties visible. Two different molecular masses were available, the characterizing parameters being listed in Table I. The volume fractions were chosen as 47:53 (h:d) because earlier experiments with a $\mathrm{H}_{2} \mathrm{O} / \mathrm{D}_{2} \mathrm{O}$ mixture with the same scattering length density led to a complete contrast matching with no Bragg peaks remaining visible.

\section{B. Small-angle neutron scattering}

To investigate the chain structure of the polymer in bulk and in confinement, SANS experiments were performed on D11 at Institut Laue-Langevin, Grenoble, France. This 40$\mathrm{m}$-instrument potentially covers a scattering vector range $Q=3 \times 10^{-4} \ldots 1 \AA^{-1}$. Here, we only used the range $0.01 \ldots 0.5 \AA^{-1}$ because the lowest $Q$ range was dominated by scattering from the SBA- 15 grain surfaces. All SANS measurements were carried out at $155^{\circ} \mathrm{C}$. Unfortunately, contrast matching did not work completely in the case of the polymers. Possible reasons for this are a density change of the polymer in the pores or scattering from residual unfilled pores. It is also possible that a fundamental imperfection of the matching due to the small-number statistics of polymer molecules plays a role. ${ }^{9}$ Apart from the possibility that pores remain unfilled, the possibility of excess polymer on the surface of the SBA-15 grains has to be considered. From elastic scans on a

TABLE I. Molecular masses and polydispersity indices of the polymers used in this study.

\begin{tabular}{lccccc}
\hline \hline & \multicolumn{2}{c}{$d$ component } & & \multicolumn{2}{c}{$h$ component } \\
\cline { 2 - 3 } \cline { 5 - 6 } Sample & $M_{n}(\mathrm{~g} / \mathrm{mol})$ & $p$ & & $M_{n}(\mathrm{~g} / \mathrm{mol})$ & $p$ \\
\hline PEP 6k & 5386 & 1.04 & & 6070 & 1.05 \\
PEP 50k & 52500 & 1.04 & & 46700 & 1.05 \\
\hline \hline
\end{tabular}

backscattering spectrometer, we estimate that this amounts to at most $20 \%$ of the polymer.

\section{EXPERIMENTAL RESULTS}

The SANS data of the bulk polymers could be fitted perfectly by Debye functions with the resulting radii of gyration listed in Table II as $R_{\mathrm{g} 0}$. The fits are shown in Fig. 3. In addition to the RPA correction mentioned above, a freely fitted flat background was added covering among other contributions the incoherent scattering. The $R_{\mathrm{g} 0}$ values are in good agreement with the calculation from the literature ${ }^{6,10}$ which gives $28 \AA$ and $82 \AA$ for the molecular masses used.

For the composite samples, because the attempt to completely contrast-match the polymer to the SBA-15 material failed, we described the scattering by a sum of contributions of (1) a $Q^{-4}$ scattering at lowest $Q,(2)$ Bragg scattering from the hexagonal pore lattice, and (3) the contribution by the polymer itself.

In order to determine the scattering from the pore lattice, an empty SBA-15 sample from the same batch was used. Fig. 4 shows the scattering pattern. It was described by a sum of $Q^{-4}$ scattering arising from the outer surface of the grains and seven separately fitted Gaussian peaks for the internal structure. It can be seen from the figure that the fit describes the scattering completely.

In a first attempt to fit the SANS data from the filled pores, a $Q^{-4}$ component and the empirical (seven Gaussian) function for the pore scattering were used together with a Debye function for the contained polymer. The prefactors of the matrix components were freely fitted because of the difference in scattering contrast between the filled and the empty SBA- 15 . In addition, a possible effect of the confinement on $R_{\mathrm{g}}$ was

TABLE II. Results from fits of the SANS data. Radius of gyration of the unconfined chain $R_{\mathrm{g}} 0$, force constant of the DK model $d$, and effective cylinder radius of the DK model $R_{\text {cyl }}$.

\begin{tabular}{lccc}
\hline \hline Sample & $R_{\mathrm{g} 0}(\AA)$ & $d(\AA)$ & $R_{\mathrm{cyl}}(\AA)$ \\
\hline PEP 6k & $29.88 \pm 0.02$ & $25.4 \pm 0.6$ & $41.7 \pm 0.8$ \\
PEP 50k & $83.73 \pm 0.07$ & $16.68 \pm 0.07$ & $42.6 \pm 0.1$ \\
\hline \hline
\end{tabular}

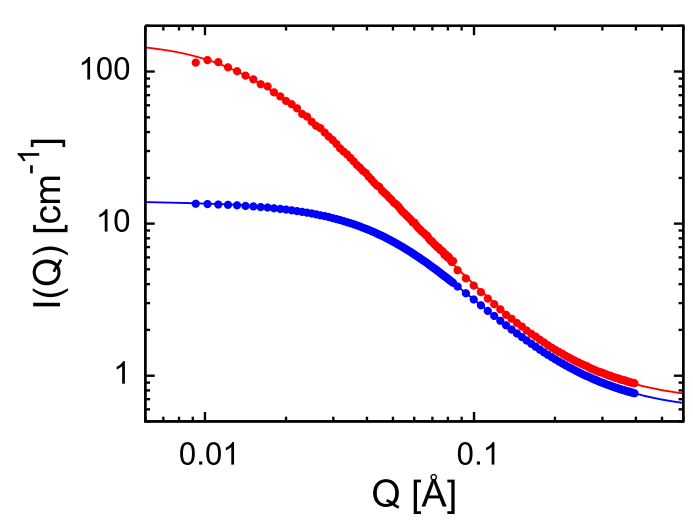

FIG. 3. SANS measurements of the bulk PEP melt. Red circles correspond to the $50 \mathrm{k}$ sample and blue circles to $6 \mathrm{k}$. Error bars would be smaller than the symbols. The curves of the same color are the respective fits with a Debye function and corrections as explained in the text. 


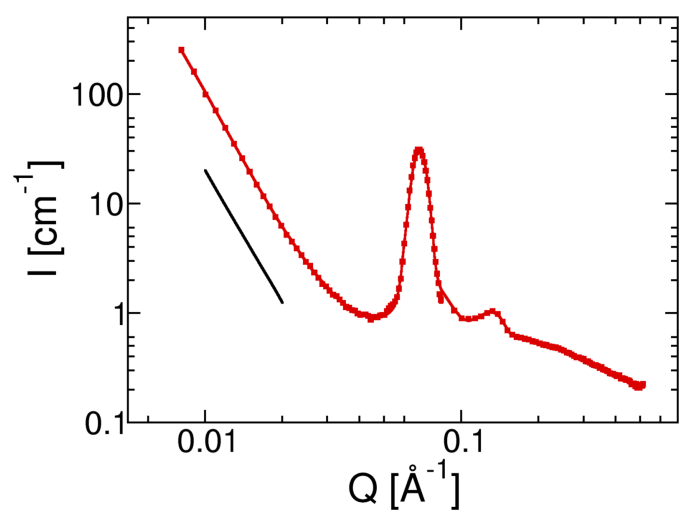

FIG. 4. SANS measurements of the empty SBA-15. The empirical fit function is explained in the text. The black line indicates the slope of the $Q^{-4}$ component visible at low $Q$.

considered by leaving this parameter free too. Despite that flexibility, the fit was not satisfactory, as can be seen from the dashed lines in Fig. 5. Especially, for the larger molecular mass (PEP 50k), a significant deviation occurs in the range $Q=0.03 \ldots 0.2 \AA^{-1}$. The corresponding fit for PEP $6 \mathrm{k}$ shows less deviation, indicating that this is due to an effect of the confinement on the chain structure.

Therefore, we replaced the Debye function by the DK expression (16) together with (19) and (20). For this fit, $R_{\mathrm{g} 0}$ was adopted from the bulk fit and only $d$ is taken as a free parameter. The continuous curves in Fig. 5(a) show that this fit describes the data much better for PEP 50k. This becomes especially clear from the inset showing the $Q$ region corresponding to $2.5 \ldots 6$ in Fig. 1. Fig. 6 shows the components of the fit function demonstrating that the polymer contribution is dominant in the range where the improvement by using DK is visible.

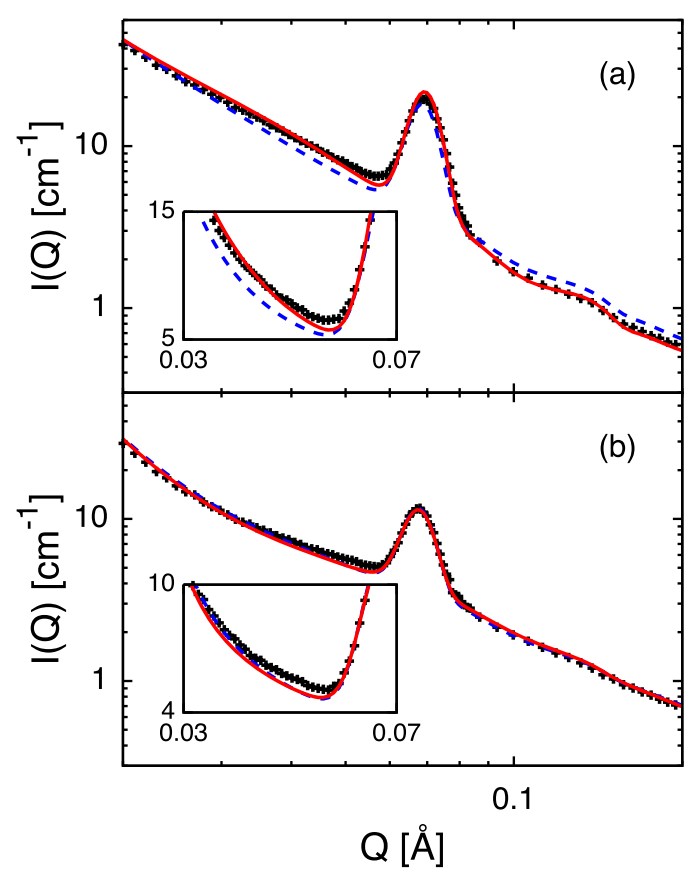

FIG. 5. SANS measurements of PEP in SBA-15. (a) PEP 50k and (b) PEP 6k. The fits consist of a sum of matrix and polymer scattering where the latter is described by the Debye model (blue dashed curve) or the Dolgushev-Krutyeva model (red continuous curve). The insets show an enlargement of the region $Q=0.03 \ldots 0.07 \AA^{-1}$.

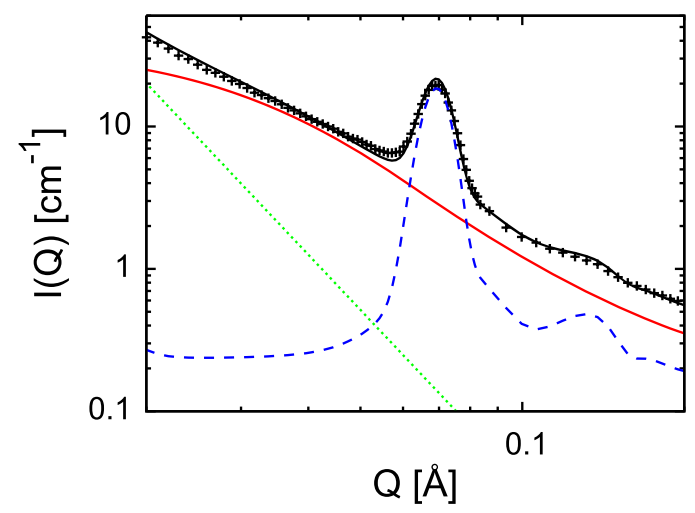

FIG. 6. Composition of the fit function for the SANS data. As an example, the fit of PEP 50k with the DK expression and without partial Debye contribution is shown. Black continuous curve: total fit function, black symbols: SANS data, red continuous curve: DK expression, blue dashed curve: pore scattering, green dotted line: $Q^{-4}$ component.

For the lower molecular mass, Fig. 5(b), there is only a faint difference between the two fits. But it has to be noted that also in that case the fit results in a "reasonable" $d$ in the sense explained below. For both molecular weights, there is a residual discrepancy between the fits and the data whose origin is unclear.

As Table II shows, the $d$ value resulting from the DK fits is smaller than the pore radius and depends on the polymer used. But as explained above, we do not expect a direct relation between $d$ and the confinement size. Indeed, using expression (23) it turns out that for both samples the radius of an equivalent cylinder is the same within error margins and agrees within $10 \%$ with that from electron microscopy.

In order to check whether this result depends on a contamination by residual bulk material scattering, we fitted the SANS data also with an additional $20 \%$ contribution of an unchanged Debye function with the bulk parameters. It turns out that with this assumption the values for $d$ are lower, $23.8 \AA$ and $10.8 \AA$ for PEP 6k and PEP 50k, respectively. While for PEP 6k this would result in a better agreement with the electron microscope cylinder radius $\left(R_{\mathrm{cyl}}=40 \AA\right)$, for PEP $50 \mathrm{k}$ there would be an $18 \%$ discrepancy $\left(R_{\text {cyl }}=32 \AA\right)$.

From Fig. 5 and the values in Table II, it is clear that for a visual demonstration of the confinement effect on the structure factor it is necessary to have a strong confinement with $R_{\text {confinement }} / R_{\mathrm{e}} \lesssim 0.2$. This may be the reason why many studies (e.g., Ref. 11) fail to see any effect of the confinement and find that the structure factor is a Debye function also in the confinement.

\section{CONSEQUENCES FOR DYNAMICS}

Because the DK model is able (and was devised) to evaluate the dynamical properties of a confined chain, clearly, it would be desirable to test the model in this aspect too. The method of choice for such a test would be neutron spin echo spectroscopy (NSE) because it is able to measure the intermediate scattering function directly and in the appropriate time range. The ability to perform such tests on the undisturbed Rouse model and the reptation model has been demonstrated in numerous experiments. ${ }^{12}$ 
We first discuss the possibility to do such a test with the samples used here. PEP 50k can be immediately excluded because the molecular mass is more than ten times above the entanglement molecular mass $\left(M_{\mathrm{e}}=4200 \mathrm{~g} / \mathrm{mol}\right) .{ }^{13}$ Although such an experiment would be interesting by itself, this would not be a test of the DK model because the DK model assumes unentangled chains. A hypothetical experimental result for PEP 6k, which is only slightly entangled, is shown in Fig. 7. Note that the experimental result would only be present in the NSE range which for a standard experiment is $10 \mathrm{ps} . .200 \mathrm{~ns}$. The $Q$ range corresponds to that of common experiments on polymers, roughly matching the polymer size. A temperature of $250{ }^{\circ} \mathrm{C}$ was chosen resulting in $W l^{4}=4.3 \times 10^{4} \AA^{4} \mathrm{~ns}^{-1} \cdot{ }^{14}$ It can be seen that only at the two lowest $Q$ values, a discernible difference between the DK model and the unmodified Rouse model exists. For reasons of chemical stability, it is not possible to raise the temperature further to shift the relaxations into the experimental window. But even if this were possible, the main problem is that for such low $Q$ values the motion of the chain is dominated by centerof-mass diffusion. The dotted curves show this for both models (obtained by neglecting all terms besides $D t$ ). The similarity with the full calculation shows that both models collapse to diffusion of an "effective" particle with friction $N \zeta$. This means that the information about the internal motions of the polymer chain is not included at such low $Q$ values. Therefore, such a test would not be convincing regarding the question whether the DK model describes the (internal) polymer motion correctly.

It is clear that, in order to see the difference in the internal modes, it is necessary to work with a stronger confinement, i.e., larger $R_{\mathrm{e}} / d$. Fig. 8 shows the result expected for $R_{\mathrm{e}}=90 \AA$, $d=10 \AA, W l^{4}=6.2 \times 10^{4} \AA^{4} \mathrm{~ns}^{-1}$, and $Q=0.1 \AA^{-1}$. There is a clear difference between the Rouse and the DK model around $10 \mathrm{~ns}$. This could not be attributed to a difference in the center-of-mass diffusion because as such it is not visible in the pure-diffusion curves (dotted) at that time. Therefore an experimental confirmation of the DK model under these conditions would be a strong evidence that it predicts the behavior of the internal chain modes correctly.

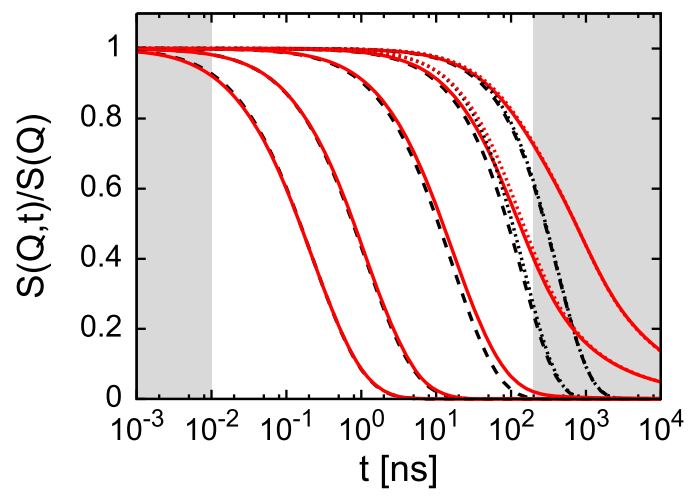

FIG. 7. Predicted result of a neutron spin echo experiment on PEP 6k. The red curves show the dynamic structure factor calculated from the DK model. The black, dashed curves show the Rouse model for comparison. The dotted curves (same colors as before) show the respective center-of-mass diffusion. The $Q$ values are $0.03,0.05,0.1,0.2$, and $0.3 \AA^{-1}$ (right to left). The experimentally inaccessible ranges are grayed out.

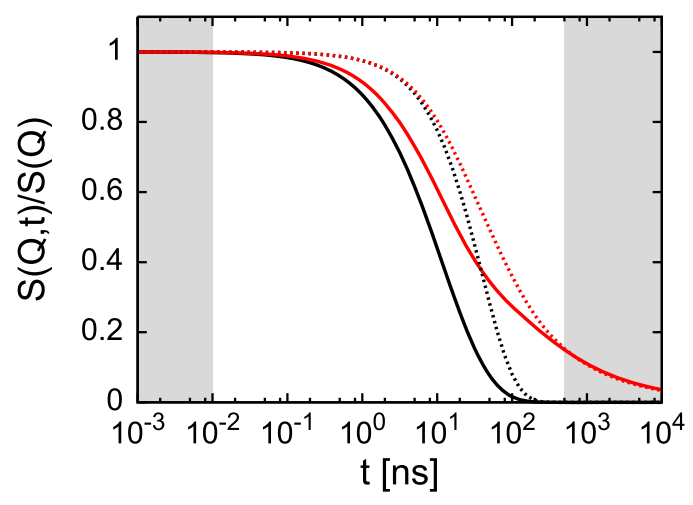

FIG. 8. Predicted result of a neutron spin echo experiment with improved conditions as described in the text. The red curves show the dynamic structure factor calculated from the DK model. The black, dashed curves show the Rouse model for comparison. The dotted curves (same colors as before) show the respective center-of-mass diffusion.

The question is now whether this experiment would be feasible. To achieve the parameters of Fig. 8 poly (dimethylsiloxane) (PDMS) would be a good candidate with $M=19000 \mathrm{~g} / \mathrm{mol}$. At that molecular mass the chains are still unentangled ${ }^{15}$ and $R_{\mathrm{e}}$ has the comparatively large value of $90 \AA$. The large value $W l^{4}$ is possible because PDMS is highly thermally stable and the experiment could be performed at 550 $\mathrm{K}$. The value of $6.2 \times 10^{4} \AA^{4} \mathrm{~ns}^{-1}$ at this temperature results from extrapolating the literature value ${ }^{16}$ at $472 \mathrm{~K}$ via the Williams-Landel-Ferry relation. ${ }^{17}$ Furthermore, in Fig. 8 we assume that a state-of-the-art NSE experiment can go up to $500 \mathrm{~ns}$ if one pushes the limits. (This would also enable to see the transition to 1D diffusion in the DK model but not be essential for the test.) The main problem lies in finding a matrix material with a sufficiently small confinement size. Fortunately, $d=10 \AA$ is not that size but instead the result of calculation (23) which is $23 \AA$ here. This is rather demanding but could be realizable by zeolites or metal-organic frameworks (MOFs).

From the calculations above, it is clear that to see an effect of the internal dynamics of a polymer chain a ratio $R_{\text {confinement }} / R_{\mathrm{e}}$ in the order of 0.2 or less is necessary. It is surprising that in a study ${ }^{18}$ partially based on NSE data ${ }^{15}$ an agreement with the DK model was found. In the NSE experiment, oriented pores with a radius of $130 \AA$ were used and a polymer with $R_{\mathrm{e}}=85 \AA$. So the situation would be worse than the one calculated in Fig. 7 and no effect could be expected. It is not clear why an agreement with the DK model is found in Ref. 18 even with a very low confinement parameter $d=5 \AA$ (converted to the units here) corresponding to $R_{\text {cyl }}=15 \AA$ which is nearly an order of magnitude lower than the actual pore size. One possible explanation would be that the strong interaction with the walls (intended by the experimenters) superimposes the "pure" confinement effect. But it also has to be noted that in the interpretation of Ref. 18 the isotropically averaged formula (16) was used while the system used in the experiment ${ }^{15}$ had oriented pores.

\section{SUMMARY AND CONCLUSIONS}

In conclusion, we can say that the Dolgushev-Krutyeva model describes the modifications of a polymer chain induced by a confinement well concerning its static properties. This 
is insofar surprising as the confining model potential is simply harmonic. Therefore the polymer density in the model is a bell-shaped function of the distance from the axis. In contrast it is more like a boxcar function in reality. It seems that the static structure factor of a confined chain does not depend strongly on the confining potential but only on its length scale. Concerning the test of the DK model regarding the dynamics, we can state that its parameters have to be well-chosen. On one hand, the model reduces to Brownian single particle dynamics in a potential for certain parameter combinations. On the other hand, entanglement effects will superimpose the confinement effect for large chains. Nevertheless, a suitable combination of parameters can be found and may be realizable in a practical experiment. The main challenge will be to find a matrix material with sufficiently narrow pores.

${ }^{1}$ M. Muthukumar, "Polymers under confinement," in Advances in Chemical Physics, edited by S. A. Rice and A. R. Dinner (John Wiley \& Sons, New York, 2012), pp. 129-196, Vol. 149.

${ }^{2}$ K. F. Freed, J. Dudowicz, E. B. Stukalin, and J. F. Douglas, "General approach to polymer chains confined by interacting boundaries," J. Chem. Phys. 133, 094901 (2010).

${ }^{3}$ M. Dolgushev and M. Krutyeva, "Coherent dynamic structure factor of a polymer chain confined into a harmonic radial potential," Macromol. Theory Simul. 21, 565 (2012).

${ }^{4}$ M. Doi and S. F. Edwards, The Theory of Polymer Dynamics (Clarendon Press, Oxford, 1984)

${ }^{5}$ R. Zorn, "Closed-form expression for the form factor of a polymer under strong confinement," Macromol. Theory Simul. 23, 84 (2014).

${ }^{6}$ A. Zitzelsberger, "Polymerkonformation in nanokompositen," M.S. thesis, University of Regensburg, 2010.

${ }^{7}$ D. Zhao, J. Feng, Q. Huo, N. Melosh, G. H. Fredrickson, B. F. Chmelka, and

G. D. Stucky, "Spherical periodic mesoporous organosilicas (sph-pmos):
Syntheses, characterisation and application in chromatography," Science 279, 548 (1998).

${ }^{8}$ V. Rebbin, "Spherical periodic mesoporous organosilicas (sph-PMOs): Syntheses, characterisation and application in chromatography," Ph.D. thesis, Justus-Liebig-Universität, Gießen, 2006.

${ }^{9}$ A. Banc, A.-C. Genix, C. Dupas, M. Sztucki, R. Schweins, M.-S. Appavou, and J. Oberdisse, "Origin of small-angle scattering from contrast-matched nanoparticles: A study of chain and filler structure in polymer nanocomposites," Macromolecules 48, 6596 (2015).

${ }^{10}$ A. Zirkel, D. Richter, W. Pyckhout-Hintzen, and L. J-Fetters, “Temperature dependence of the unperturbed dimensions of alternating poly(ethylenepropylene)," Macromolecules 25, 954-960 (1992).

${ }^{11}$ J. Martin, M. Krutyeva, M. Monkenbusch, A. Arbe, J. Allgaier, A. Radulescu, P. Falus, J. Maiz, C. Mijangos, J. Colmenero, and D. Richter, "Direct observation of the transition from free to constrained single-segment motion in entangled polymer melts," Phys. Rev. Lett. 90, 058302 (2003).

${ }^{12}$ D. Richter, M. Monkenbusch, A. Arbe, and J. Colmenero, Neutron Spin Echo in Polymer Systems (Springer, Berlin, 2005).

${ }^{13}$ A. Wischnewski, M. Monkenbusch, L. Willner, D. Richter, and G. Kali, "Direct observation of confined single chain dynamics by neutron scattering," Phys. Rev. Lett. 104, 197801 (2010).

${ }^{14}$ D. Richter, B. Farago, R. Buters, L. J. Fetters, J. S. Huang, and B. Ewen, "On the origins of entanglement constraints," Macromolecules 26, 795-804 (1993).

${ }^{15}$ M. Krutyeva, A. Wischnewski, M. Monkenbusch, L. Willner, J. Maiz, C. Mijangos, A. Arbe, J. Colmenero, A. Radulescu, O. Holderer, M. Ohl, and D. Richter, "Effect of nanoconfinement on polymer dynamics: Surface layers and interphases," Phys. Rev. Lett. 110, 108303 (2013).

${ }^{16}$ H. Götz, B. Ewen, G. Meier, and M. Monkenbusch, "Neutron scattering investigations on the statics and dynamics of polydimethyl- and polyethylmethylsiloxane melts," Macromol. Chem. Phys. 202, 3334 (2001).

${ }^{17}$ J. D. Ferry, Viscoelastic Properties of Polymers (John Wilex \& Sons, New York, 1980).

${ }^{18}$ J.-M. Y. Carrillo and B. G. Sumpter, "Structure and dynamics of confined flexible and unentangled polymer melts in highly absorbing cylindrical pores," J. Chem. Phys. 141, 074904 (2014). 